\title{
Múltiples dientes supernumerarios en un paciente pediátrico no sindrómico: Una condición rara
}

\author{
Multiple supernumerary teeth in a non-syndromic pediatric patient: A rare condition \\ Sandro Alexander Lévano Loayza ${ }^{1 a, b}$, Miguel Benjamín Perea Paz ${ }^{2 c, ~ d, 3 e ~}$
}

\section{RESUMEN}

La presencia de múltiples dientes supernumerarios en la cavidad bucal es una alteración del desarrollo dental infrecuente en pacientes que no tienen un síndrome asociado, estos pueden inducir diversas alteraciones como maloclusiones, atraso en la erupción dentaria, reabsorción de los dientes adyacentes, formación de quistes, dilaceraciones radiculares y giroversiones. El presente caso clínico tiene como objetivo reportar la presencia de múltiples dientes supernumerarios, que ocasionaron la erupción ectópica y giroversión de piezas dentarias adyacentes, provocando una mordida cruzada en la cavidad oral de un paciente pediátrico no sindromico del Centro Dental Docente Cayetano Heredia en el 2019. El tratamiento realizado fue la extracción simple de los dientes supernumerarios erupcionados y la extracción compleja con colgajo de los retenidos.

PALABRAS CLAVE: Diente supernumerario, anomalías dentarias, patología.

\section{SUMMARY}

The presence of multiple supernumerary teeth in the oral cavity is an infrequent alteration of dental development in patients who do not have an associated syndrome, that can induce various alterations such as malocclusions, delay in dental eruption, reabsorption of adjacent teeth, cyst formation, root dilacerations and giroversions. The objective of this clinical case is to report the presence of multiple supernumerary teeth, which caused the ectopic eruption and gyroversion of adjacent teeth, causing a crossbite in the oral cavity of a non-syndromic pediatric patient of the Centro Dental Docente Cayetano Heredia in 2019. The treatment performed was the simple extraction of the erupted supernumerary teeth and the complex extraction with flap of the retained ones.

KEY WORDS: Supernumerary teeth, tooth abnormalities, pathology.

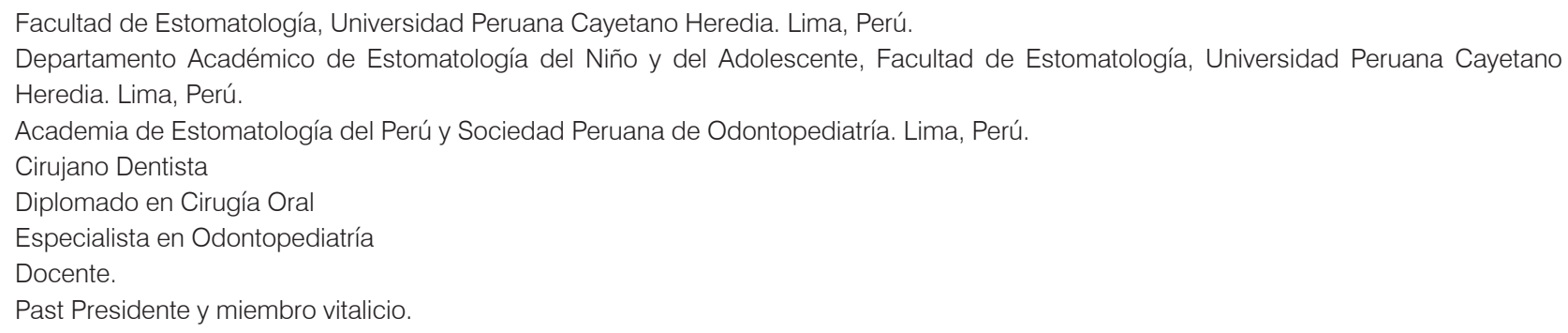




\section{INTRODUCCIÓN}

La prevalencia de los dientes supernumerarios (SN) oscila entre un $0,4 \%$ hasta un $3,8 \%$ en la población mundial $(1,2)$. Su ubicación es variada, pero en la mayoría de casos se localizan en la línea media superior, recibiendo el nombre de mesiodens. Cuando estos se presentan en la región molar se denominan paramolares, si se presentan por detrás de la tercera molar reciben el nombre de distomolares y los parapremolares son los que se ubican entre los premolares. Además de ello pueden presentarse de forma cónica, rudimentaria, tuberculada y suplementaria, similar a los dientes temporales o permanentes. También se pueden clasificar de acuerdo a su proceso de erupción de manera retenida, impactada y erupcionada (3-6).

Actualmente la etiología de los dientes SN aún no es clara, por lo que se basa en teorías como la dicotomía del folículo dental, filogenética, factor genético y la hiperactividad de la lámina dental, siendo esta ultima la más aceptada (7-9).

El diagnóstico se puede realizar mediante un examen clínico y exámenes radiográficos y dependiendo del caso se realiza el tratamiento más adecuado teniendo en cuenta la indicación ortodóntica, localización anatómica, morfología y angulación dentaria, relación con estructuras vecinas, estado de erupción, edad, conducta y estado sistémico del paciente y sobre todo la patología que este presentando en ese momento como maloclusiones, atraso en la erupción dentaria, reabsorción de los dientes adyacentes, formación de quistes, dilaceraciones radiculares y giroversiones (10-13).

La presencia de múltiples dientes $\mathrm{SN}$ es frecuente en individuos que presentan diversos síndromes como la Displasia Cleidocraneal, Síndrome de Gardner, Down, Fabry-Anderson, Hallermann-Streiff, etc. Sin embargo, se ha registrado algunos casos en los cuales la presencia de estos no estaba asociado a ningún síndrome (14-18).

El presente caso clínico tiene como objetivo reportar la presencia de múltiples dientes supernumerarios, que ocasionaron la erupción ectópica y giroversión de piezas dentarias adyacentes, provocando una mordida cruzada en la cavidad oral de un paciente pediátrico no sindromico del Centro Dental Docente Cayetano Heredia en el 2019. El tratamiento realizado fue la extracción simple de los dientes supernumerarios erupcionados y la extracción compleja con colgajo de los retenidos.

\section{REPORTE DEL CASO}

Paciente masculino de 12 años de edad sin antecedentes sistémicos, fue referido al Servicio de Odontopediatría del Centro Dental Docente de la Universidad Peruana Cayetano Heredia, por un odontólogo particular, quien en su evaluación clínica halló dientes supernumerarios en el sector anterosuperior. Los antecedentes médicos personales y familiares, con la evaluación extraoral no sugirieron sospecha de la presencia de algún síndrome o trastorno que pueda estar asociado a la presencia de estos supernumerarios. Debido a que no se evidencio hipoplasia facial, aplasia clavicular, quistes epidérmicos, pólipos intestinales, osteomas, braquicefalia, retraso mental, angioqueratomas, opacidad corneal, hipotricosis ni acondroplasia, que son signos comunes en síndromes relacionados a pacientes con múltiples dientes $\mathrm{SN}$.

A la evaluación intraoral se evidenció la presencia de dos dientes supernumerarios, el primero fue un $\mathrm{SN}$ fusionado entre las piezas dentarias 1.1 y 1.2 y el segundo se encontró entre la 2.1 y 2.2. Además, se observó erupción ectópica de los incisivos centrales y caninos de la maxila, mordida cruzada anterior y posterior del lado derecho (figura 1). Se decidió la toma de una radiografía panorámica y periapicales, se halló tres supernumerarios retenidos entre las piezas 3.3 y 3.5 , otro por distal de la pieza 3.8 y dos más entre las piezas 4.3 y 4.5 (figura 2). Por lo tanto, se procedió a realizar una interconsulta al Servicio de Ortodoncia y Ortopedia Maxilar quien indicó realizar las exodoncias de todos los dientes supernumerarios, con la excepción del distomolar, más los primeros premolares de cada maxilar, debido a la discrepancia alveolo dentaria negativa de $-19 \mathrm{~mm}$ en la maxila y de $-4.5 \mathrm{~mm}$ en la mandíbula.

Para la planificación del tratamiento quirúrgico se indicó la toma de una Tomografía Computarizada de Haz Cónico (TCHC) para el abordaje de los dientes retenidos; luego se realizaron las exodoncias simples, sin mayor dificultad y las exodoncias complejas por encontrarse por detrás de las raíces de los premolares inferiores tuvieron una mayor dificultad quirúrgica, estas se realizaron por cuadrantes bajo anestesia local y de forma ambulatoria (figura 3). Posterior a cada 
procedimiento quirúrgico simple se le medicó al paciente solo con Paracetamol de 500mg cada 6 horas por 3 días, y para los procedimientos quirúrgicos complejos AINES y un antibiótico (Ketorolaco de 30 mg una hora después de la intervención, Naproxeno de $275 \mathrm{mg}$ cada 8 horas por 5 días y Amoxicilina de $500 \mathrm{mg}$ cada 8 horas por 6 días). Se realizaron controles clínicos y retiro de suturas a los 7 días de cada procedimiento quirúrgico y se observó heridas quirúrgicas a nivel de lecho alveolar en proceso de cicatrización normal y favorable. Fue dado de alta por el Servicio de Odontopediatría y posteriormente se realizó la transferencia al Servicio de Ortodoncia y Ortopedia Maxilar para el tratamiento de la maloclusión.
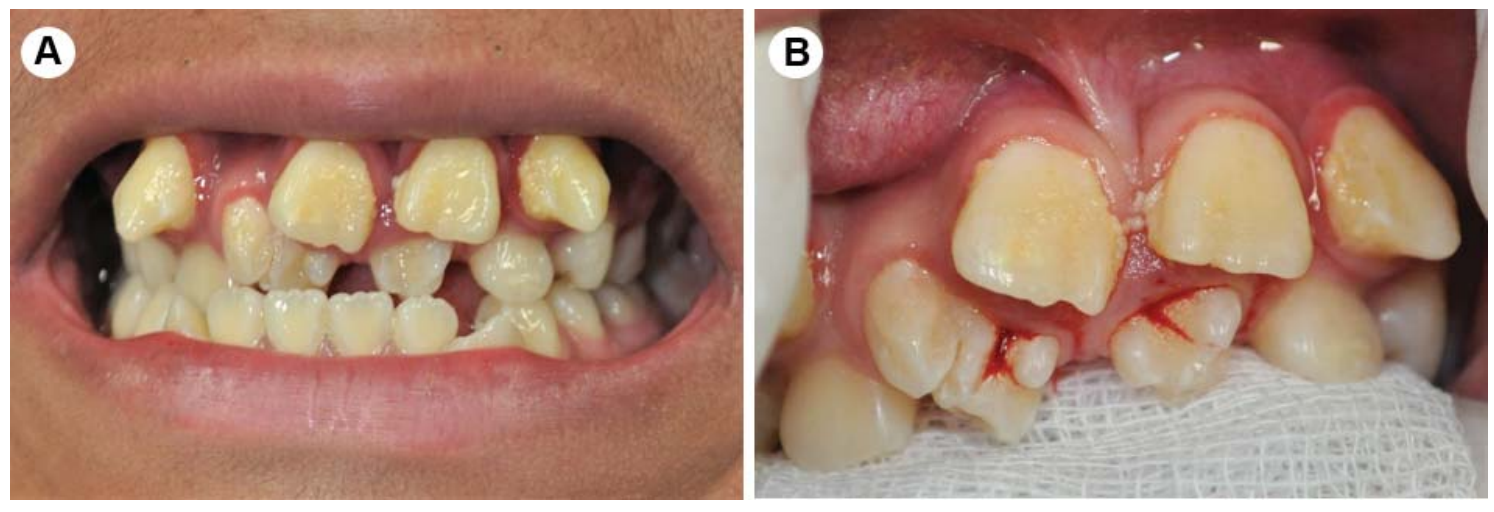

Figura 1. Fotografías clínicas intraorales. A, B: Vista frontal. Dientes SN entre piezas dentarias $1.1 \mathrm{y}$ $1.3,2.1$ y 2.3 . Erupción ectópica de piezas $1.1,1.3,2.1$ y 2.3, mordida cruzada anterior y posterior del lado derecho.
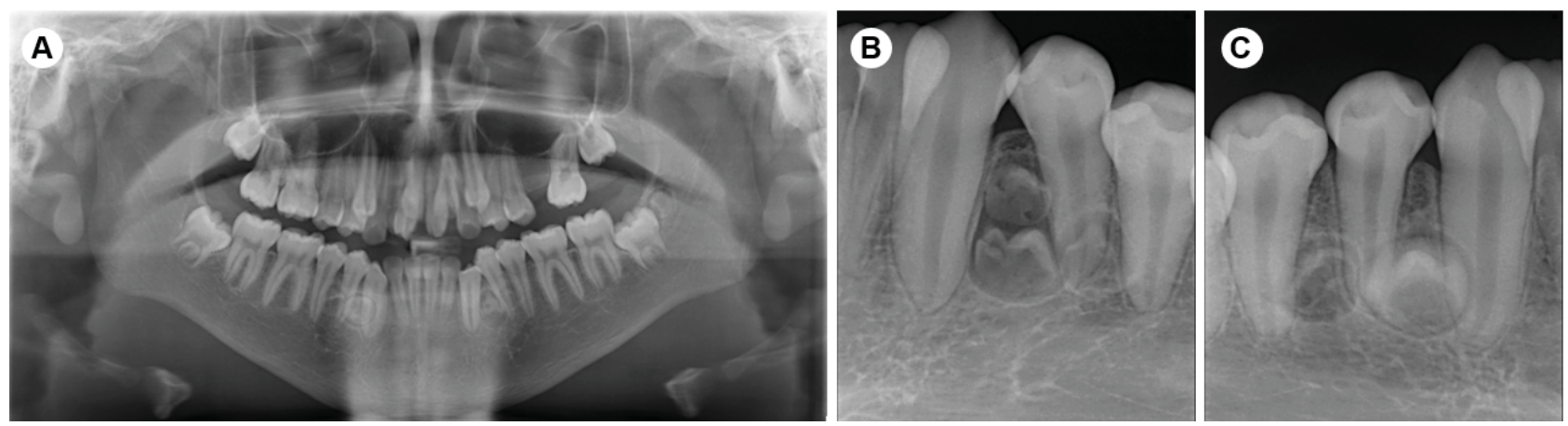

Figura 2. Radiografías. A: Panorámica. B: Periapical del Cuadrante III (3 dientes SN parapremolares). C: Periapical del Cuadrante IV (2 dientes SN parapremolares). 

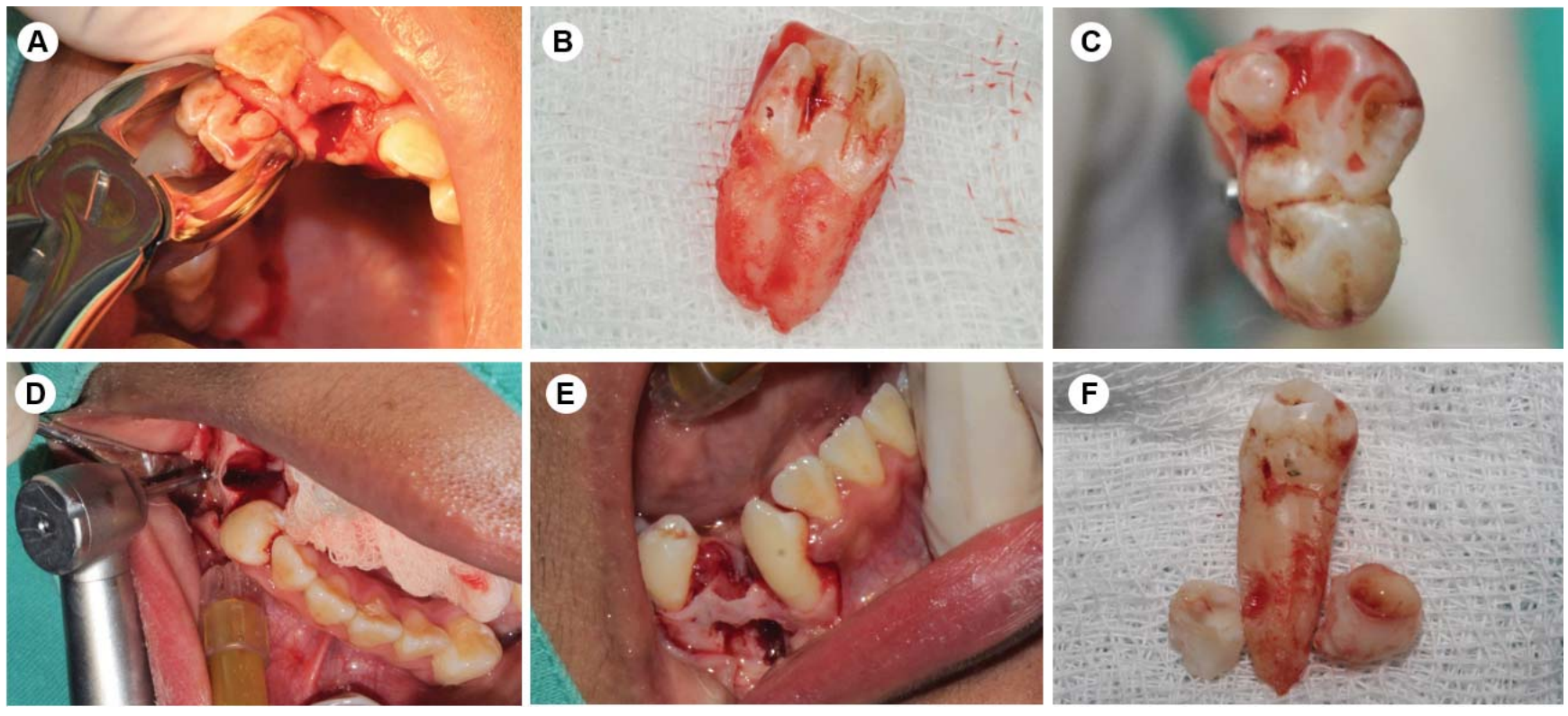

Figura 3. A: Prensamiento con fórceps del diente SN fusionado. B: Vista lateral de diente SN fusionado. C: Vista de la corona de diente SN fusionado. D: Decolado mucoperióstico y osteotomía (post exodoncia de pieza dentaria 4.4). E: Cavidad ósea de diente SN. F: Dientes SN parapremolares y pieza dentaria 4.4.

\section{DISCUSIÓN}

El término utilizado para describir la presencia de dientes SN es hiperdoncia; según Fardi y Bäckman et al., tiene una prevalencia de 0,1 a 3,8\% en dentición permanente y de $0,35-0,6 \%$ en la decidua $(19,20)$. De acuerdo a Cortés-Bretón et al., quienes hicieron una revisión de múltiples dientes $\mathrm{SN}$ en trece pacientes con cincuenta y cinco dientes supernumerarios, definiendo múltiple como la presencia de tres o más en un solo paciente, además señalando que la prevalencia de múltiples dientes $\mathrm{SN}$ en pacientes no sindromicos es de menos del $1 \%$ siendo reforzada por varios autores (21), resaltando así la condición rara del presente caso.

El presente caso es de un paciente masculino con múltiples dientes $\mathrm{SN}$ en ambos maxilares y su distribución; dos en la pre maxila y cinco en la región premolar y uno en retromolar. McBeain et al., mencionan que la incidencia de los dientes supernumerarios es mayor en pacientes masculinos, lo cual coincide con el presente caso clínico. También menciona que la localización anatómica más frecuente de hallar dientes supernumerarios es en la maxila, especialmente en la zona de los incisivos (5).
Sin embargo, Ansari et al., reportaron un caso de 13 SN siendo la región premolar la más afectada (22), coincidiendo con nuestro caso.

Los dientes SN del presente caso en su mayoría retenidos, ocasionaron varias alteraciones en la oclusión del paciente. Pérez et al., determinaron que, según el tipo de erupción de los dientes supernumerarios, el de mayor frecuencia fue el retenido (erupción interrumpida de germen dentario en edad de erupción) seguido del impactado (erupción detenida de diente por barrera física o malposición de esta). En el presente caso clínico hubo la presencia de 8 dientes supernumerarios, de los cuales 6 de estos eran retenidos y solo 2 de estos eran erupcionados, coincidiendo con lo anteriormente mencionado. También mencionan que la complicación más frecuente por la presencia de estos, es la malposición dentaria de los dientes adyacentes (18) lo cual concuerda con nuestro caso ya que la presencia de estos tanto en el maxilar como en la mandíbula ocasionaban malposiciones dentarias de los dientes contiguos.

Existen dos posturas sobre el momento adecuado 
para la realización de la intervención quirúrgica que involucra la exodoncia de dientes SN. Siendo la primera decisión la exodoncia de estos tan pronto como hayan sido diagnosticados, la segunda es dejarlos hasta que se desarrollen las raíces de los dientes adyacentes, para evitar posibles daños colaterales. Patchett et al., señalan que la edad promedio ideal debe ser de nueve años y cuatro meses para que el diente supernumerario pueda ser quirúrgicamente extraído (23). En nuestro caso el paciente había sobrepasado la edad de nueve años cuando acudió al servicio por tratamiento los dientes adyacentes a los dientes supernumerarios estaban completamente desarrollados, por lo cual no hubo ninguna contraindicación en realizar el acto quirúrgico una vez que fueron diagnosticados.

\section{CONCLUSIONES}

La presencia de múltiples dientes supernumerarios no siempre está asociada a un síndrome, sin embargo, el cirujano dentista debe conocer cuáles son los principales síndromes asociados además de sus características principales para la remisión del paciente al servicio especializado.

La presencia de dientes supernumerarios en el exa men clínico intraoral, indica la necesidad de tomar una radiografía panorámica por la posibilidad de encontrar más dientes supernumerarios dentro de los maxilares.

El examen clínico radiográfico debe ser minucioso y los tratamientos quirúrgicos deben ser bien evaluados y planificados, ya que el éxito de los mismos permite evitar complicaciones.

\section{Correspondencia:}

Sandro Lévano Loayza

Correo electrónico: sandro.levano.1@gmail.com

\section{REFERENCIAS BIBLIOGRAFICAS}

1. Liu M, Yang X, Lv K, Li Z. Minimally-invasive alternative to the extraction of deeply-impacted supernumerary teeth using a computer-designed surgical template. British J Oral and Maxillofac Surg. 2019; 57(1):285-287.

2. Oropeza M. Dientes supernumerarios. Reporte de un caso clínico. Rev Odon Mex. 2013; 17(2): 91-96.
3. He D, Mei L, Wang Y, Li J, Li H. Association between maxillary anterior supernumerary teeth and impacted incisors in mixed dentition. JADA. 2017; 148(8):595-603.

4. Jammal N, Silva R. Prevalence of supernumerary teeth in a mexican sample. Rev Mexicana Ortodoncia. 2015; 3(2):88-91.

5. McBeain M, Miloro M. Characteristics of supernumerary teeth in nonsyndromic population in an urban dental school setting. J Oral Maxillofac Surg. 2018; 76(1):933-938.

6. Celikoglu M, Kamak H, Oktay H. Prevalence and characteristics of supernumerary teeth in a non-syndrome Turkish population: Associated pathologies and proposed treatment. Med Oral Patol Oral Cir Bucal. 2010; 15(4):575-8.

7. Açikgöz A, Açikgöz G, Tunga, U, Otan F. Characteristics and prevalence of non-syndrome multiple supernumerary teeth: a retrospective study. Dentomaxillofac Radio. 2006; 35:185-90.

8. Mukhopadhyay S. Mesiodens: a clinical and radiographic study in children. J Indian Soc Pedod Prev Dent. 2011; 29(1):34-38.

9. Gallas M, García A. Case study: retention of permanent incisors by mesiodens: a family affair. Br Dent $\mathrm{J}$. 2000; 188:63-64.

10. Blanco Ballesteros G. Dientes múltiples supernumerarios. Reporte de un caso. Revista Estomatológica. 2005; 13(1):13-18.

11. Garvey M, Barry H, Blake M. Supernumerary teeth: an overview of classification, diagnosis and management. J Can Dent Assoc. 1999; 65(11):612-616.

12. Pavoni C, Mucedero M, Lagana G, Paoloni V, Cozza P. Impacted maxillary incisors: diagnosis and predictive measurements. Ann Stomatol. 2012; 3(4): 100-105.

13. Erdody G, López JC, Quesada D. Quiste dentígero asociado a diente supernumerario. Ciencia Odontógica. $2011 ; 8(1)$ : 68-72.

14. Galal $O$. Multiple unerupted and supernumerary teeth in a patient with cleidocranial displasia. 2018; 1(3): 118-120.

15. Thomas J, Ragavi B, Raneesha P, et al. Hallermann-streiff syndrome. Indian J Dermatol. 2013; 58(5):383-384.

16. Regattieri L, Parker J. Supernumerary teeth associated with Fabry-Anderson's syndrome. Oral Surg Oral Med Oral Pathol. 1973; 35(3):432-3

17. Basaran G, Erkan M. One of the rarest syndromes in dentistry: gardner syndrome. Eur J Dent. 2008; 2(3):208-212.

18. Pérez I, Chávez K, Ponce D. Prevalence of supernumerary teeth on panoramic radiographs in a non-adult Peruvian sample. Int. J. Odontostomat. 2014; 8(3):377-383.

19. Fardi A, Kondylidou-Sidira A, Bachour Z, Parisis N, Tsirlis A. Incidence of impacted and supernumerary teeth- a radiographic study in a North Greek population. Med Oral Patol Oral Cir Bucal. 2011; 16:56-61. 
20. Bäckman B, Wahlin YB. Variations in number and morphology of permanent teeth in 7-year-old Swedish children. Int J Paediatr Dent. 2001; 11:11-7.

21. Cortés-Bretón Brinkmann J, Barona-Dorado C, Martínez- Rodriguez N, Martín-Ares M, MartínezGonzález JM. Nonsyndromic multiple hyperdontia in a series of 13 patients: Epidemiologic and clinical considerations. J Am Dent Assoc. 2012; 143:16-24.

22. Ansari AA, Malhotra S, Pandey RK, Bharti K. Nonsyndromic multiple supernumerary teeth: Report of a case with 13 supplemental teeth. BMJ Case Rep. 2013; 3(6): 11-36.

23. Patchett L, Crawford M, Cameron C, Stephens D. The management of supernumerary teeth in childhood - a retrospective study of practice in Bristol Dental Hospital, England and Westmead Dental Hospital, Sydney, Australia. Int J Paediatr Dent. 2001; 11 (4): 259-65. doi: 10.1046 / j.1365-263x.2001.00282.x

Recibido: 06-01-20

Aceptado: 13-05-20 